\title{
Detection of hyperphosphorylated tau protein and $\alpha$-synuclein in spinal cord of patients with Alzheimer's disease
}

This article was published in the following Dove Press journal:

Neuropsychiatric Disease and Treatment

26 February 2016

Number of times this article has been viewed

\author{
Yanjun Guo ${ }^{1,2}$ \\ Luning Wang ${ }^{2}$ \\ Mingwei $\mathrm{Zhu}^{2}$ \\ Honghong Zhang ${ }^{3}$ \\ Yazhuo $\mathrm{Hu}^{3}$ \\ Zhitao $\mathrm{Han}^{3}$ \\ Jia $\mathrm{Liu}^{4}$ \\ Weiqin Zhao' \\ Dexin Wang' \\ 'Department of Neurology, Beijing \\ Friendship Hospital, Capital Medical \\ University, ${ }^{2}$ Department of Geriatric \\ Neurology, PLA General Hospital, \\ ${ }^{3}$ Institute of Geriatrics, Chinese PLA \\ General Hospital \& Chinese PLA \\ Medical Academy, ${ }^{4}$ Department of \\ Neurology, Xuanwu Hospital, Capital \\ Medical University, Beijing, People's \\ Republic of China
}

Correspondence: Luning Wang Department of Geriatric Neurology, PLA General Hospital, 28 Fuxing Road, Haidian District, Beijing 100853, People's Republic of China

$\mathrm{Tel}+86$ I3 33II $3880 \mid$

$\mathrm{Fax}+86$ 0I 063139807

Emaillnw301@।63.com
Abstract: The aim of this study was to investigate the neuropathological features of the spinal cord in patients suffering with Alzheimer's disease (AD). Spinal cord tissue collected from three $\mathrm{AD}$ patients and eight controls was selected for the study. Data were collected at T2, T8, T10, L4, and S2 spinal levels. The sections were subjected to hematoxylin and eosin and Gallyas-Braak staining methods and then were immunostained with antibodies such as phosphorylated tau protein (AT8), $\alpha$-synuclein, A $\beta$, amyloid precursor protein , ubiquitin, and TDP-43. Pathological changes exhibited by the biomarkers were detected by microscopy. Neurofibrillary tangles (NFTs) were detectable in spinal anterior horn motor neurons in two of the three AD patients. AT8-positive axons or axon-like structures and AT8 expression in glial cells were detected in all three AD cases. Hyperphosphorylation of tau protein was detected in spinal anterior horn cells, glial cells, and axons, and its severity was associated with NFTs in the brain tissue. $\alpha$-Synuclein-positive Lewy bodies and scattered Lewy-like neuritis were detected in the medial horn of the thoracic spinal cord and ventral sacral gray matter, respectively, in one patient who had AD with Lewy bodies. Neither amyloid deposition nor amyloid precursor protein and TDP-43 expression was detected in the spinal cord of AD patients. Spinal cord of AD patients was observed to contain phosphorylated tau protein and $\alpha$-synuclein immunoreactive structures, which may play a role in dyskinesia and autonomic dysfunction in advanced AD.

Keywords: Alzheimer's disease, $\alpha$-synuclein, tau protein, neurofibrillary tangles, histopathology

\section{Introduction}

Clinical manifestation and pathological changes of neurodegerative diseases have overlap, which include the main pathogenic protein in different parts of different diseases. Although cognitive disorder is the main clinical manifestation associated with Alzheimer's disease (AD), movement and autonomic dysfunctions are very common. ${ }^{1}$ In addition to movement disorders in Parkinson's disease, dementia can still be observed, and Lewy bodies (LB) appear in both the spinal cord and in the sympathetic trunk ganglia and also between the heart and the rectum muscle plexus. Research concerning the regions outside the brain tissue of AD patients, particularly with regard to spinal cord pathology, should be strengthened in order to understand the symptoms associated with cognitive impairment of the movement system and its pathogenesis in $\mathrm{AD}$ patients. de Calignon et $\mathrm{al}^{2}$ studied a transgenic mouse model and found that tau pathology progresses from transgene-expressing neurons in the entorhinal cortex to neurons without detectable transgene expression, beginning with the neighboring cells of entorhinal cortex and followed by propagation to neurons downstream in the 
synaptic circuit. Liu et $\mathrm{al}^{3}$ found that tau protein can spread trans-synaptically along the anatomically connected networks and between connected and vulnerable neurons.

There have been only a few studies on the expression of tau protein and other biomarkers of neurodegeneration in the spinal cord. A study using an animal model of Tg30tau mouse with AD showed that NFT changes in the spinal cord and brain stem occurred prior to those in the forebrain, ${ }^{4}$ and recent studies also revealed that impaired axonal transportation is an important pathogenesis observed in neurodegenerative diseases, ${ }^{5}$ and phosphorylated tau (p-tau) protein, ubiquitin, and amyloid precursor protein (APP) are the main components of axonal transportation. Another study found that TDP-43 inclusions are not only expressed in the spinal cord in amyotrophic lateral sclerosis but are also present in the AD, Pick's disease, and other diseases. ${ }^{6}$ However, the pathogenesis of movement disorders in the AD patients requires further study.

Based on the clinical and experimental studies mentioned earlier, we hypothesized that pathological changes involving motor neurons and the autonomic nervous system may occur in the spinal cord of patients suffering with AD. The aim of this investigation was to study the expression of the biomarkers associated with neurodegenerative diseases in the spinal cord of AD patients.

\section{Materials and methods General clinical characteristics of study subjects}

The study included three male patients suffering with $\mathrm{AD}$ and whose age ranged from 85 to 96 years (mean: $89.33 \pm 5.89$ years). Eight subjects served as controls, five had other nervous system diseases (OND group) and three had no severe neurological diseases (two comprised the old control [OC] group and one comprised the middle-aged control [MC] group). Among the controls, seven were male and one was female, and their mean age was $78.86 \pm 8.51$ years. The age difference between the AD patients and the controls was not significant $(P=0.092)$. The time interval among the AD patients and controls from death to autopsy was $28.66 \pm 18.04$ and $30.50 \pm 13.86$ hours, respectively, and the difference was not significant $(P=0.371)$. There were no significant differences in sex, age, and education between the AD patients and controls. Autopsy was done after written informed consent was obtained from the patients family. Both the autopsy and the study were approved by the ethics committee of PLA General Hospital, Peoples Republic of China.

The cause of death and central nervous system (CNS) pathology observed in the AD patients and controls are
Table I Cause of death and CNS pathology

\begin{tabular}{|c|c|c|c|c|c|}
\hline $\begin{array}{l}\text { Case } \\
\text { group }\end{array}$ & $\begin{array}{l}\text { Case } \\
\text { no }\end{array}$ & Sex & $\begin{array}{l}\text { Age } \\
\text { (years) }\end{array}$ & $\begin{array}{l}\text { Cause of } \\
\text { death }\end{array}$ & $\begin{array}{l}\text { Central nervous } \\
\text { system pathology }\end{array}$ \\
\hline \multirow[t]{3}{*}{$\begin{array}{l}\text { AD } \\
\text { group }\end{array}$} & 1 & Male & 88 & $\begin{array}{l}\text { Myocardial } \\
\text { infarction }\end{array}$ & $\begin{array}{l}\text { AD; cerebral } \\
\text { infarction } \\
\text { with spotting } \\
\text { hemorrhage }\end{array}$ \\
\hline & 2 & Male & 96 & $\begin{array}{l}\text { OSCC } \\
\text { metastasis }\end{array}$ & $\begin{array}{l}A D \text { with Lewy } \\
\text { bodies }\end{array}$ \\
\hline & 3 & Male & 85 & Lung infection & $A D$ \\
\hline \multirow[t]{5}{*}{$\begin{array}{l}\text { ONDs } \\
\text { group }\end{array}$} & 4 & Female & 73 & $\begin{array}{l}\text { Cerebral } \\
\text { embolism }\end{array}$ & Cerebral infarction \\
\hline & 5 & Male & 76 & Lung cancer & $\begin{array}{l}\text { Delayed } \\
\text { radioencephalopathy }\end{array}$ \\
\hline & 6 & Male & 89 & $\begin{array}{l}\text { Cerebral } \\
\text { hernia }\end{array}$ & $\begin{array}{l}\text { Traumatic brain } \\
\text { injury }\end{array}$ \\
\hline & 7 & Male & 81 & Lung cancer & $\begin{array}{l}\text { Meningeal } \\
\text { carcinomatosis }\end{array}$ \\
\hline & 8 & Male & 65 & Lung infection & $\begin{array}{l}\text { Subacute cerebral } \\
\text { hemorrhage }\end{array}$ \\
\hline OC & 9 & Male & 73 & Heart failure & Lacunar infarction \\
\hline group & 10 & Male & 84 & $\begin{array}{l}\text { Respiratory } \\
\text { failure }\end{array}$ & Lacunar infarction \\
\hline $\begin{array}{l}\text { MC } \\
\text { group }\end{array}$ & 11 & Male & 49 & $\begin{array}{l}\text { Liver } \\
\text { transplantation }\end{array}$ & None \\
\hline
\end{tabular}

Abbreviations: CNS, central nervous system; AD, Alzheimer's disease; ONDs, other nervous system diseases; OC, old control; MC, middle-aged control; OSCC, oral squamous cell carcinoma.

presented in Table 1. All the three AD patients presented with dementia, memory loss, and the loss of computing ability; two had mental disorders; two had hallucinations; one had confabulation; two showed personality changes; one had delusion of killing; and two had disorientation. One patient had no aphasia, limb paralysis, or sensory abnormalities but presented with bilateral pathological positive (case 1) and another had bowel and bladder dysfuction (case 2). There are no reports with regard to the walking disorders or other autonomic nervous system lesions (Table 2).

Autopsy specimens of brain and spinal cord were obtained from the Tissue Bank of the Institute of Geriatrics, PLA General Hospital, Beijing, People's Republic of China. After reviewing the clinical histories and pathology reports, we selected three definite $\mathrm{AD}$ patients based on Khachaturian criteria, the criteria of the Consortium to Establish A Registry for Alzheimer's Disease (CEARD), ${ }^{7}$ and Braak-Braak grading criteria. ${ }^{8}$

\section{Preparation of brain and spinal cord tissues}

Conventional autopsies were conducted, and brain and spinal cord were harvested and drop-fixed in $10 \%$ formalin for $2-4$ weeks. The sampling process of brain tissues was performed in accordance with the programs adopted by brain libraries of Massachusetts General Hospital of Harvard 


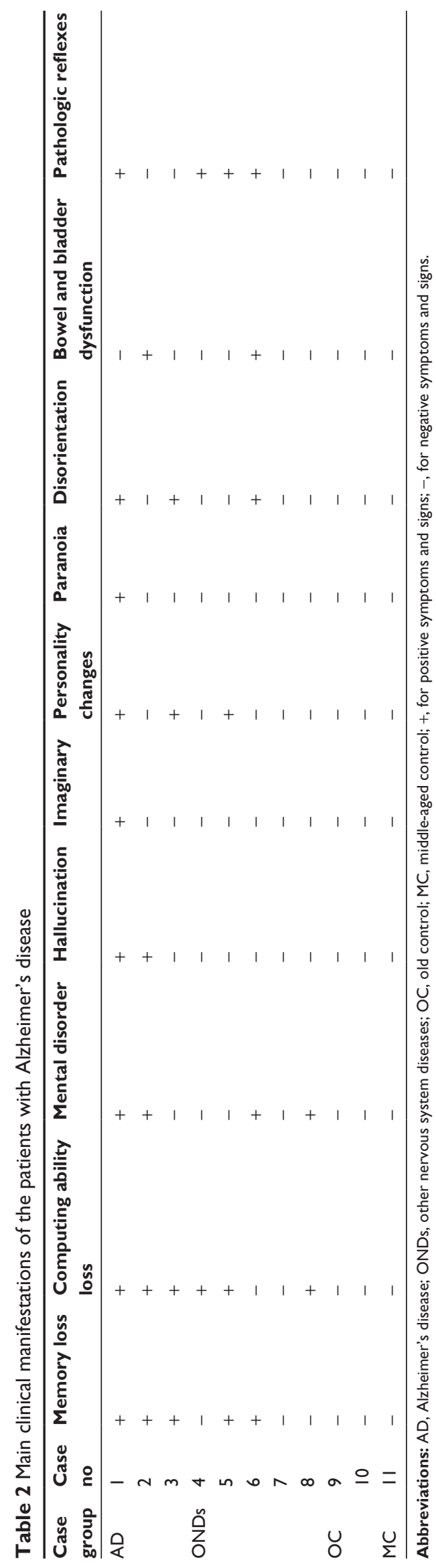

University and Austria. Spinal cords were harvested from the T2, T8, T10, L4, and S2 segments (cervical spinal cord was not harvested in this group). After conducting conventional dehydration treatments (Automatic Rotary Dehydrator 4634; Cherry, Japan), paraffin embedding (Paraffin Embedding Machine EG1140H; Leica Microsystems, Wetzlar, Germany), and continuous slicing with a thickness of $7 \mu \mathrm{m}$ (Slicer RM2135; Leica), the slices were divided into eight sets and were treated with hematoxylin and eosin (H\&E) and GallyasBraak stains according to the method described by Braak et al. ${ }^{9}$ The slices were also subjected to immunohistochemical cytochemical stainings using six antibodies: anti-A $\beta$, AT8, anti-synuclein, anti-ubiquitin, anti-APP, and anti-TDP-43.

Sections were dewaxed with water, incubated in $3 \%$ peroxide for 15 minutes, and washed with distilled water. Sections were stained to determine the presence of p-tau using mouse monoclonal antibody AT8 (1:200; Innogenetics, Ghent, Belgium) without antigen retrieval. Sections were stained to determine the presence of repaired A $1-42$ (AntiHuman $\beta$-Amyloid (1-42): mouse anti-human monoclonal antibody, 1:200; Dako, Glostrup, Denmark) with formic acid for 3 minutes for epitope exposure. Sections were initially treated with primary antibody agents such as rabbit anti-human $\alpha$-synuclein polyclonal antibody (1:800; Sigma-Aldrich, St Louis, MO, USA) or rabbit anti-human ubiquitin polyclonal antibody (1:200; Dako) or rabbit anti-human APP C-terminal polyclonal antibody (1:2,000; Sigma) or rabbit anti-human TDP-43 polyclonal antibody (1:1,500; Proteintech Group, Inc., Chicago, IL, USA) and were placed in a humid chamber and kept overnight at $4^{\circ} \mathrm{C}$. Then they were washed with phosphatebuffered saline (PBS), incubated with secondary antibody agents for 30 minutes, washed with PBS, and then DAB working fluid was added for color development. Then the sections were stained with H\&E stain again, followed by differentiation with $1 \%$ hydrochloride alcohol, counterstaining with anti-blue, dehydrating, clearing, and cementing with a neutral gum.

The samples were analyzed using an Olympus BX60 fluorescence microscope.

\section{Results}

\section{Neuropathological changes in brain tissue of $A D$ patients}

Examples of neuropathological changes that are characteristic of AD are shown in Figure 1A-D. Reduction of hippocampal neurons and granulovacuolar degeneration of the pyramidal cells were observed in the hippocampus CA1 area in all the three AD cases. Senile plaques were detected in the brain cortex and hippocampus, and amyloidosis of 

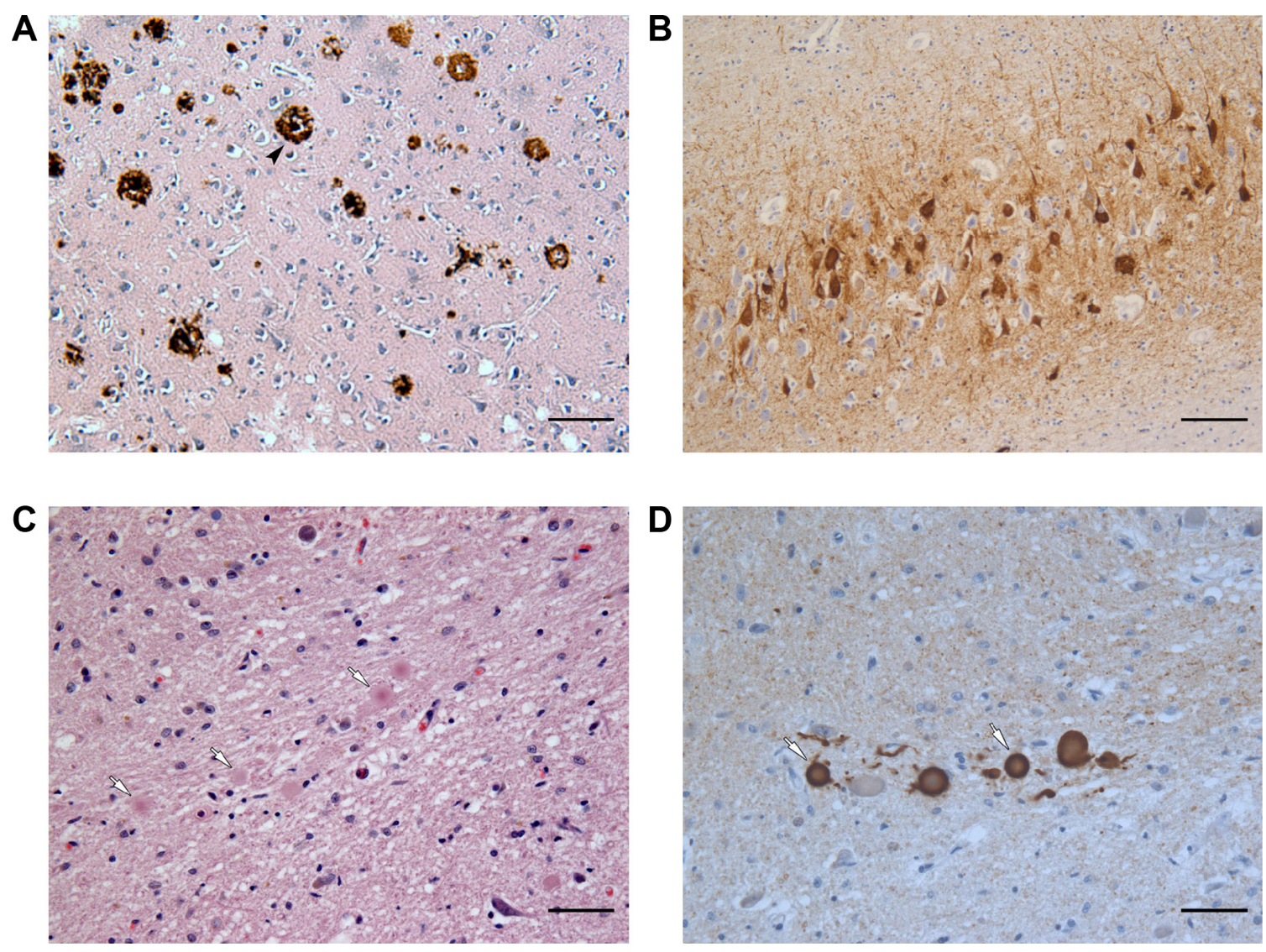

Figure I Neuropathological changes in the brains of AD patients.

Notes: (A) Immunohistochemical detection of a large number of $A \beta$ plaques in a neocortex in the frontal lobe of $A D$. Scale bar equals $100 \mu$ m; Immunohistochemical detection of a large number of senile plagues (arrow head) in the frontal lobe of $A D$ (A $\beta I-42$ immunostain). (B) AD illustrating numerous NFTs and NT in hippocampus (AT8). Scale bar equals $100 \mu \mathrm{m}$. (C) Lewy bodies (white arrows) in the dorsal nucleus of vagus nerve in medulla in case 2 (H\&E staining). Scale bar equals $200 \mu \mathrm{m}$. (D) Lewy bodies (white arrows) in the dorsal nucleus of vagus nerve of medullary in case 2 ( $\alpha$-synuclein). Scale bar equals $100 \mu \mathrm{m}$.

Abbreviations: AD, Alzheimer's disease; NFTs, neurofibrillary tangles; NT, neuropil threads; H\&E, hematoxylin and eosin.

small vessels was detected under the pia mater and within the parenchyma in AD patients (Figure 1A). NFTs and neuropil threads (NT) were detected in a large number of neurons in the cortex and hippocampus regions (Figure 1B). $\mathrm{A} \beta$ deposition was observed on the vessel wall of the brain parenchyma, showing amyloid angiopathy changes. The $\alpha$-synuclein staining of the brain tissue from case 2 showed a relatively larger number of LB and Lewy neuritis (LN) in the dorsal motor nucleus of the vagus nerve and red nucleus (Figure 1C and D). Specific expressions of TDP-43 were not observed in all the three AD cases.

\section{Neuropathological changes in spinal cord of AD patients}

HE staining showed the morphology of the anterior horn cells of the spinal cord and there was no significant difference in the number of the horn cells between the $\mathrm{AD}$ and control groups.

Gallyas-Braak-positive neurons and corresponding axons were detected in the ventral horn of lumbar and thoracic spinal cord in all the AD patients (Figure 2A and B). In cases 1 and 2, Gallyas-Braak-positive axons were observed in each plane.

AT8 immunohistochemistry staining results revealed AT8 positive expression in the spinal cords of all $3 \mathrm{AD}$ patients (Table 3). 1) In cases 1 and 2, NFT changes were detected in anterior horn cells at the L4 spinal surface, and NFTs were observed to be thickened uniformly or with varied thicknesses and were disorderly arranged (Figure 2C and D). 2) Spinal white matter revealed axonal degeneration in all the three cases (Figure 2E) and presented with AT8 expression in the glial cells, particularly in the anterior funiculi and spinal anterior joints at the spinal surfaces (Figure 3A). However, in the control group, we did not observe NFTs or AT8 expression in the glial cells, except for AT8-positive scattered structures in the spinal T10 plane in case 6, who is in the OND group.

We did not observe A $\beta$ or APP expression in the gray matter and vasculature of each spinal section in both the $\mathrm{AD}$ and control groups.

In case 2, we observed LB (Figure 3B) in the intermediolateral column of lateral horn in T10 spinal cord, and 

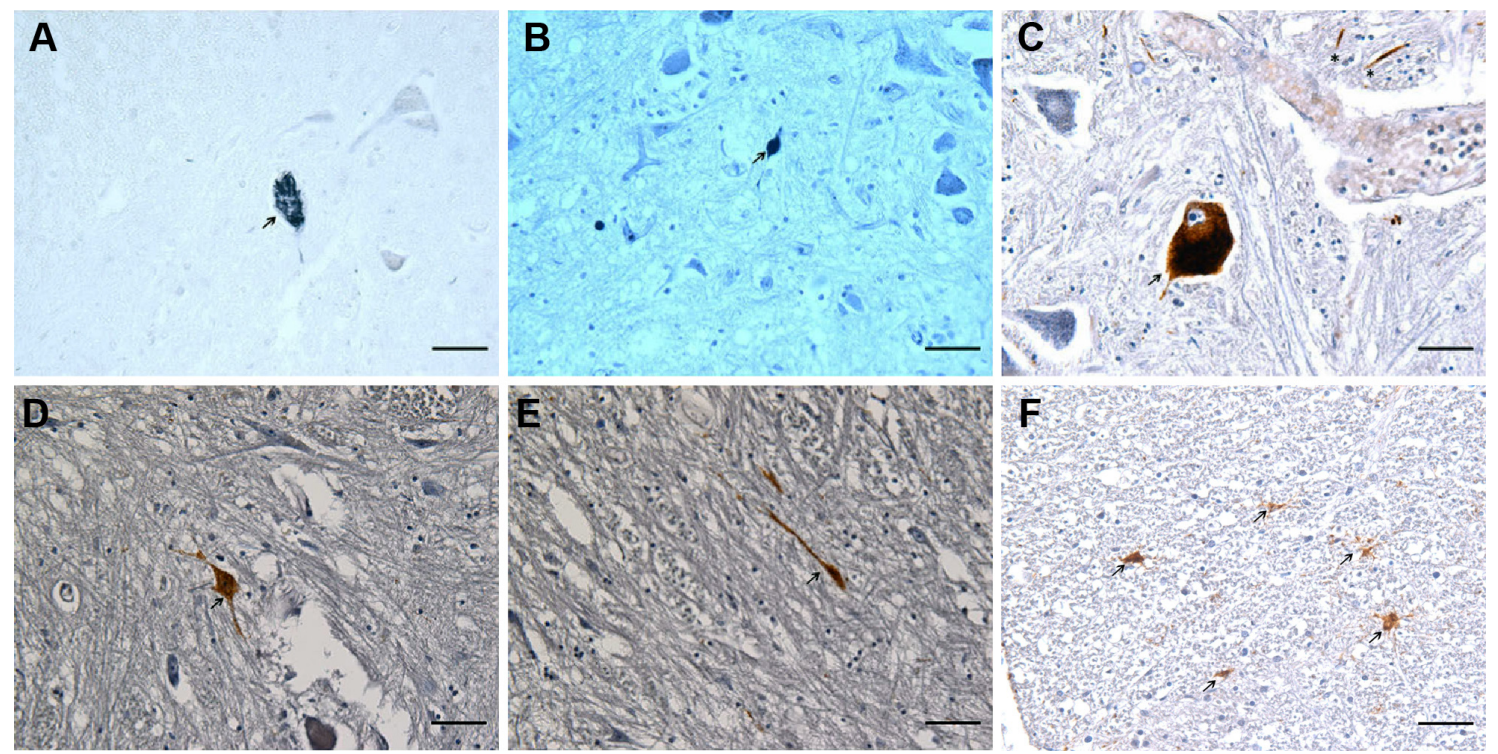

Figure 2 Expression of hyperphosphorylated tau protein in spinal cord in $A D$.

Notes: (A) Shows Gallyas-Braak positive neurons (arrow) and axons on spinal S2 surface in AD case (Gallyas-Braak staining). Scale bar equals $50 \mu \mathrm{m}$. (B) Shows NFTs (arrow) in anterior horn cells on spinal L4 surface (Gallyas-Braak staining). Scale bar equals $50 \mu \mathrm{m}$. (C) Shows NFTs (arrow) and NTs (*) in spinal anterior horn on spinal L4 surface in AD (case 2) (AT8-immunostain). Scale bar equals $50 \mu \mathrm{m}$. (D) Shows NFTs (arrow) and axon in anterior horn cells on spinal L4 surface in AD (case I) (AT8immunostain). Scale bar equals $50 \mu \mathrm{m}$. (E) Reveals AT8-positive axons (arrow) on spinal L4 surface in AD (case I) (AT8-immunostain). Scale bar equals $50 \mu \mathrm{mm}$. (F) Reveals relatively large amount of glial cells expressing AT8 (arrows) in the spinal anterior joint on the spinal 14 surface in case 2 (AT8-immunostain). Scale bar equals $50 \mu \mathrm{m}$. Abbreviations: AD, Alzheimer's disease; NFTs, neurofibrillary tangles; NT, neuropil threads.
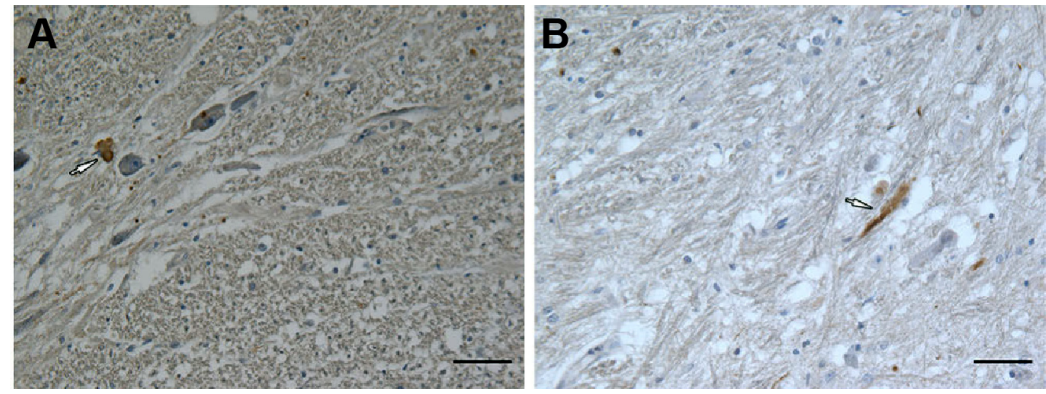

Figure 3 Expression of $\alpha$-synuclein in spinal cord in $A D$.

Notes: $(\mathbf{A})$ Lewy bodies (white arrow) in the intermediate lateral thoracic spinal cord ( $\alpha$-synuclein-immunostain) in AD with Lewy bodies (case 2 ). Scale bar equals $50 \mu$ m. (B) Illustrates lewy neuritis (white arrow) in sacral spinal cord of case 2 ( $\alpha$-synuclein-immunostain). Scale bar equals $50 \mu \mathrm{m}$.

Abbreviation: AD, Alzheimer's disease.

Table 3 Expression of NFTs in AD patients

\begin{tabular}{|c|c|c|c|c|c|c|c|c|c|c|c|c|c|c|c|c|c|c|}
\hline $\begin{array}{l}\text { Case } \\
\text { group }\end{array}$ & $\begin{array}{l}\text { Case } \\
\text { no }\end{array}$ & $\begin{array}{l}\text { Braak-Braak } \\
\text { stage }\end{array}$ & T2 & T8 & TIO & L4 & S2 & DMN & $\mathbf{R N}$ & LC & SN & Amyg & Hippo & $F$ & $\mathbf{P}$ & $\mathbf{T}$ & 0 & $\overline{\mathrm{Ce}}$ \\
\hline \multirow[t]{3}{*}{$A D$} & I & $\mathrm{V}-\mathrm{VI}$ & 0 & 0 & 0 & 2 & I & I & 0 & $I$ & I & 4 & 4 & 2 & 2 & 3 & 2 & 0 \\
\hline & 2 & V-VI & 0 & 0 & I & 2 & 0 & 3 & 0 & I & 1 & 4 & 4 & 2 & 2 & 3 & 2 & 0 \\
\hline & 3 & III-IV & 0 & I & I & 0 & 0 & NA & NA & NA & NA & 3 & 3 & I & 2 & 2 & I & 0 \\
\hline \multirow[t]{5}{*}{ ONDs } & 4 & 1 & 0 & 0 & 0 & 0 & 0 & 0 & 0 & 0 & 0 & 0 & 1 & 0 & 1 & I & 0 & 0 \\
\hline & 5 & I & 0 & 0 & 0 & 0 & 0 & 0 & 0 & 0 & 0 & 0 & 1 & 0 & 0 & 0 & 0 & 0 \\
\hline & 6 & I-II & 0 & 0 & I & 0 & 0 & 0 & 0 & 0 & 0 & I & I & 0 & 0 & I & 0 & 0 \\
\hline & 7 & 1 & 0 & 0 & 0 & 0 & 0 & 0 & 0 & 0 & 0 & 0 & I & 0 & I & I & 0 & 0 \\
\hline & 8 & I & 0 & 0 & 0 & 0 & 0 & 0 & 0 & 0 & 0 & 0 & 0 & 0 & 0 & 0 & 0 & 0 \\
\hline \multirow[t]{2}{*}{ OC } & 9 & I & 0 & 0 & 0 & 0 & 0 & 0 & 0 & 0 & 0 & 0 & I & 0 & 0 & I & 0 & 0 \\
\hline & 10 & I-II & 0 & 0 & 0 & 0 & 0 & 0 & 0 & 0 & 0 & 0 & I & 0 & 1 & I & 0 & 0 \\
\hline$M C$ & 11 & I & 0 & 0 & 0 & 0 & 0 & 0 & 0 & 0 & 0 & 0 & 0 & 0 & 0 & 0 & 0 & 0 \\
\hline
\end{tabular}

Notes: NFTs evaluation ${ }^{8}$ : adopted Braak-Braak criteria, spinal NFTs grading was divided into five grades referring to the cortex NFTs grading method $(0=$ none, $\mathrm{I}=$ rare, $2=$ a few, $3=$ mediate, $4=$ large amount).

Abbreviations: AD, Alzheimer's disease; ONDs, other nervous system diseases; OC, old control; MC, middle-aged control; DMN, dorsal nucleus of vagus nerve; RN, red nucleus; LC, locus coeruleus; SN, substantia nigra; Amyg, amygdala; Hippo, hippocampus; F, frontal lobe; P, parietal lobe; T, temporal lobe; O, occipital lobe; Ce, cerebellum; NA, not available; NFT, neurofibrillary tangles. 
Table 4 Expression of $\alpha$-synuclein in AD patients

\begin{tabular}{|c|c|c|c|c|c|c|c|c|c|c|c|c|c|c|c|c|}
\hline Case no & T2 & T8 & TIO & L4 & S2 & DMN & RN & LC & SN & Amyg & Hippo & $\mathbf{F}$ & $\mathbf{P}$ & $\mathbf{T}$ & 0 & $\mathrm{Ce}$ \\
\hline I & 0 & 0 & 0 & 0 & 0 & 0 & 0 & 0 & 0 & 0 & 0 & 0 & 0 & 0 & 0 & 0 \\
\hline 2 & 2 & 2 & 2 & 0 & 2 & 3 & 2 & I & 0 & 0 & 0 & 0 & 0 & 0 & 0 & 0 \\
\hline 3 & 2 & 2 & 2 & 0 & I & NA & NA & NA & NA & NA & NA & 0 & 0 & 0 & 0 & 0 \\
\hline 4 & 0 & 0 & 0 & 0 & 0 & 0 & 0 & 0 & 0 & 0 & 0 & 0 & 0 & 0 & 0 & 0 \\
\hline 5 & 0 & 0 & 0 & 0 & 0 & 0 & 0 & 0 & 0 & 0 & 0 & 0 & 0 & 0 & 0 & 0 \\
\hline 6 & 0 & 0 & 0 & 0 & 0 & 0 & 0 & 0 & 0 & 0 & 0 & 0 & 0 & 0 & 0 & 0 \\
\hline 7 & 0 & 0 & 0 & 0 & 0 & 0 & 0 & 0 & 0 & 0 & 0 & 0 & 0 & 0 & 0 & 0 \\
\hline 8 & 0 & 0 & 0 & 0 & 0 & 0 & 0 & 0 & 0 & 0 & 0 & 0 & 0 & 0 & 0 & 0 \\
\hline 9 & 0 & 0 & 0 & 0 & 0 & 0 & 0 & 0 & 0 & 0 & 0 & 0 & 0 & 0 & 0 & 0 \\
\hline 10 & 0 & 0 & 0 & 0 & 0 & 0 & 0 & 0 & 0 & 0 & 0 & 0 & 0 & 0 & 0 & 0 \\
\hline 11 & 0 & 0 & 0 & 0 & 0 & 0 & 0 & 0 & 0 & 0 & 0 & 0 & 0 & 0 & 0 & 0 \\
\hline
\end{tabular}

Notes: $\alpha$-synuclein evaluation criteria ${ }^{20} 0=$ None, $I=$ mild (scattered LBs or LNs), $2=$ mediate (at least one $L B$ or few $L N$ in low power vision), $3=$ severe (at least four LB and/or scattered $L N$ in low power vision), $4=$ very severe (numerous $L B$ and/or numerous $L N$ ).

Abbreviations: AD, Alzheimer's disease; DMN, dorsal nucleus of vagus nerve; RN, red nucleus; LC, locus coeruleus; SN, substantia nigra; Amyg, amygdala; Hippo, hippocampus; F, frontal lobe; P, parietal lobe; T, temporal lobe; O, occipital lobe; Ce, cerebellum; NA, not available; LB, Lewis bodies; LN, Lewy neuritis.

dispersed LN was encountered in the intermediolateral column of lateral horn in T2, T5, and T10 spinal cord and the anterolateral column of ventral gray in sacral cord of Case 2 (Table 4).

No characteristic ubiquitin inclusions were detected in the spinal cord of all the three cases suffering with AD. TDP-43-positive inclusions in the neuronal and glial cells of spinal cord in the $\mathrm{AD}, \mathrm{OC}$, and $\mathrm{MC}$ groups were also not detected.

\section{Discussion}

We observed hyperphosphorylated tau protein in the spinal anterior horn cells, glial cells, and axons in the three patients suffering with $\mathrm{AD}$. In the $\mathrm{AD}$ cases, NFTs in the brain cortex were in the Braak stage V-VI (Braak-Braak grades), and AT8 expression was observed in neurons, glial cells, and axons of the spinal cord. While in the control group, we did not observe NFTs or AT8 expression in the glial cells, except for AT8-positive scattered structure in the spinal T10 plane in one case.

The spinal cord is in communication with the brain as well as the peripheral nerves and therefore is the logical place to look for disease processes that manifest in both the regions. An increasing body of literature indicates the presence of disease-specific spinal cord histopathology in multiple neurodegenerative diseases, including amyotrophic lateral sclerosis, Parkinson's disease, and other motor neuron diseases. However, for $\mathrm{AD}$, the most common neurodegenerative disease, there have been relatively few published works on spinal cord histopathology. There are only five published studies on tau expression in the spinal cords of AD and normal aging subjects. ${ }^{10-14}$ Wang et al using the Gallyas silver stain, and reported that among a series of centenarians, NFTs and/or NT in the spinal cord were present in $9 / 10$ cases with a clinicopathologic diagnosis of $\mathrm{AD}$, while 7/9 non-demented (ND) cases were positive for these findings, predominantly within the cervical subdivision. ${ }^{11}$ Saito et al revealed tau- and/ or Gallyas-positive structures within the spinal cord of all the eleven AD subjects as well as in 7/10 normal controls, and densities were observed to be higher in the anterior horn. ${ }^{12}$ Dugger et al investigated, using immunohistochemical methods, the presence of $\mathrm{p}$-tau in the spinal cord of 46 cases with a clinicopathological diagnosis of $\mathrm{AD}$ as well as in $37 \mathrm{ND}$ individuals lacking any defined CNS-related clinicopathological diagnosis. Meanwhile, they found cervical cord segments to be the most frequently affected region ( $96 \% \mathrm{AD}$ vs $43 \% \mathrm{ND})$, followed by thoracic ( $69 \%$ AD vs $37 \%$ ND), lumbar $(65 \%$ $A D$ vs $27 \% \mathrm{ND}$ ), and sacral ( $53 \%$ AD vs $13 \% \mathrm{ND}$ ) regions. Previous literature on NFTs and abnormal p-tau within the human spinal cord is relatively sparse, but the studies indicated that spinal cord p-tau-immunoreactive pathology has important implications for both the pathogenesis and clinical manifestations of $\mathrm{AD} .{ }^{14}$

An animal study using the Tg30tau mouse that bears the two pathogenic mutations of P301S and G272V revealed that NFTs changes of the spinal cord and brain stem preceded those of the forebrain and a more severe early-stage lesion was found in the spinal cord and brain stem. ${ }^{4}$ During the early course of $\mathrm{AD}$, abnormal tau accumulates in the entorhinal cortex and later accumulates in the hippocampus and subsequently in the neocortex. ${ }^{3}$ It has been hypothesized that this sequential pattern can be explained by the transsynaptic spread. ${ }^{3}$ It was suggested that abnormal tau was transferred to synaptically connected neurons via release of abnormal tau at the synapse. ${ }^{3}$ The same trans-synaptic spread mechanism possibly involving a prion-like cascade 
might explain our findings of AT8 in the spinal cord of AD patients. Hyperphosphorylation of tau protein is known to be involved in axonal transport stabilization and promotion of microtubule polymerization, and it participates in the transport of vesicles and organelles from axons to synaptic terminals. It was also reported that tau overexpression affects axonal transport by obstructing kinesin movement on microtubules, human tau is likely released from terminals and taken up by glia as the axons degenerate. ${ }^{2,3,5,15}$ Our study found that AT8 expression in some astrocytes in anterior commissure of the spinal cord suggests that axonal transport barriers cause hyperphosphorylation of tau protein at the axon terminals.

We did observe characteristic expression of $\alpha$-synucleinpositive structures in the spinal cord of case 2 , the patient diagnosed to have $\mathrm{AD}$ with $\mathrm{LB}$. In this patient, we observed LB and scattered LN in the intermediolateral column of lateral horn in the thoracic cord, and we also noted scattered LN-like structures in the lateral anterior sacral gray matter without other pathological changes. Ding et al investigated the autopsied brains and spinal cords from 23 Japanese patients living for over 100 years and found that of the ten $\mathrm{AD}$ patients, four had LB in their brain tissues and two had positive expression of $\alpha$-synuclein both in the spinal cords and brain tissues. ${ }^{16}$ No cases with independent spinal $\alpha$-synuclein expression have been previously reported. ${ }^{17}$ In case 2 , there were $\alpha$-synuclein-positive structures in the spinal cord and brain stem, which correlated with the accumulation of $\alpha$-synuclein in his brain tissues. The reason for the bowel and bladder dysfunction in this patient was probably due to the fact that the presence of $\alpha$-synuclein-positive structures in the spinal cord might result in the occurrence of certain forms of autonomic dysfunction.

In 2006, Neumann et al ${ }^{18}$ confirmed that TDP-43 is the major protein component of frontotemporal lobar dementia with ubiquitin and ubiquitin inclusions in amyotrophic lateral sclerosis. Pathological changes of TDP-43 can be observed in approximately $20 \%-30 \%$ of the $\mathrm{AD}$ patients. ${ }^{6}$ AmadorOrtiz et al found that TDP-43 positive immunoreactivity was correlated with a higher Braak NFT stage. ${ }^{19}$

We did not observe TDP-43-positive inclusions in the spinal cord. Neither A $\beta$ nor APP deposition was found in the spinal cord.

In our study, hyperphosphorylation of tau protein was detected in the spinal anterior horn cells, glial cells, and axons in $\mathrm{AD}$ patients, and its severity was associated with the presence of NFTs in brain tissue. LB and dispersed LN were encountered in the intermediolateral column of lateral horn in the thoracic cord and the anterolateral region of ventral gray in sacral cord of AD with Lewy Bodies. Based on the results mentioned earlier, the spinal NFTs, NT, and LB in the $\mathrm{AD}$ patients may be related to movement disorders or autonomic dysfunction in advanced AD. Varying degrees and different affected areas of hyperphosphorylated tau protein, $\alpha$-synuclein, and other lesions in the spinal cord of AD patients indicate that different lesions may play a different role in the pathogenesis of $\mathrm{AD}$, which might be associated with the transsynaptic transmission caused by hyperphosphorylation of tau protein and $\alpha$-synuclein. However, the specific pathogenesis and clinical significance still requires further study. The main shortcoming of this study was a very small sample size.

\section{Acknowledgment}

This study was supported by National Key Basic Research and Development Program ("973”) (2006CB500700).

\section{Disclosure}

The authors report no conflicts of interest in this work.

\section{References}

1. Hou CE, Carlin D, Miller BL. Non-Alzheimer's disease dementias: anatomic, clinical, and molecular correlates. Can J Psychiatry. 2004; 49(3):164-171.

2. de Calignon A, Polydoro M, Suarez-Calvet M, et al. Propagation of tau pathology in a model of early Alzheimer's disease. Neuron. 2012; 73(4):685-697.

3. Liu L, Drouet V, Wu JW, et al. Trans-synaptic spread of tau pathology in vivo. PLoS One. 2012;7(2):e31302.

4. Leroy K, Bretteville A, Schindowski K, et al. Early axonopathy preceding neurofibrillary tangles in mutant tau transgenic mice. Am J Pathol. 2007;171(3):976-992.

5. Adalbert R, Gilley J, Coleman MP. Abeta, tau and ApoE4 in Alzheimer's disease: the axonal connection. Trends Mol Med. 2007; 13(4):135-142.

6. Uryu K, Nakashima-Yasuda H, Forman MS, et al. Concomitant TARDNA-binding protein 43 pathology is present in Alzheimer disease and corticobasal degeneration but not in other tauopathies. J Neuropathol Exp Neurol. 2008;67(6):555-564.

7. Morris JC, Heyman A, Mohs RC, et al. The Consortium to Establish a Registry for Alzheimer's Disease (CERAD). Part I. Clinical and neuropsychological assessment of Alzheimer's disease. Neurology. 1989;39(9):1159-1165.

8. Braak H, Braak E. Neuropathological stageing of Alzheimer-related changes. Acta Neuropathol. 1991;82(4):239-259.

9. Braak H, Braak E, Ohm T, Bohl J. Silver impregnation of Alzheimer's neurofibrillary changes counterstained for basophilic material and lipofuscin pigment. Stain Technol. 1988;63(4):197-200.

10. Yamada M. On the distribution of senile changes in the spinal cord. Folia Psychiatr Neurol Jpn. 1978;32(2):249-251.

11. Wang Y, Hashizume Y, Yoshida M, Inagaki T, Kameyama T. Pathological changes of the spinal cord in centenarians. Pathol Int. 1999; 49(2):118-124.

12. Saito Y, Murayama S. Expression of tau immunoreactivity in the spinal motor neurons of Alzheimer's disease. Neurology. 2000;55(11): 1727-1729.

13. Schmidt ML, Zhukareva V, Perl DP, et al. Spinal cord neurofibrillary pathology in Alzheimer disease and Guam Parkinsonism-dementia complex. J Neuropathol Exp Neurol. 2001;60(11):1075-1086. 
14. Dugger BN, Hidalgo JA, Chiarolanza G, et al. The distribution of phosphorylated tau in spinal cords of Alzheimer's disease and nondemented individuals. J Alzheimers Dis. 2013;34(2):529-536.

15. Sahara N, Murayama M, Higuchi M, Suhara T, Takashima A. Biochemical distribution of tau protein in synaptosomal fraction of transgenic mice expressing human P301L tau. Front Neurol. 2014;5:26.

16. Ding ZT, Wang Y, Jiang YP, etal. Characteristics of alpha-synucleinopathy in centenarians. Acta Neuropathol. 2006;111(5):450-458.

17. Bloch A, Probst A, Bissig H, Adams H, Tolnay M. Alpha-synuclein pathology of the spinal and peripheral autonomic nervous system in neurologically unimpaired elderly subjects. Neuropathol Appl Neurobiol. 2006;32(3):284-295.
18. Neumann M, Sampathu DM, Kwong LK, et al. Ubiquitinated TDP-43 in frontotemporal lobar degeneration and amyotrophic lateral sclerosis. Science. 2006;314(5796):130-133.

19. Amador-Ortiz C, Lin WL, Ahmed Z, et al. TDP-43 immunoreactivity in hippocampal sclerosis and Alzheimer's disease. Ann Neurol. 2007; 61(5):435-445.

20. McKeith IG, Dickson DW, Lowe J, et al. Diagnosis and management of dementia with Lewy bodies: third report of the DLB Consortium. Neurology. 2005;65(12):1863-1872.

\section{Publish your work in this journal}

Neuropsychiatric Disease and Treatment is an international, peerreviewed journal of clinical therapeutics and pharmacology focusing on concise rapid reporting of clinical or pre-clinical studies on a range of neuropsychiatric and neurological disorders. This journal is indexed on PubMed Central, the 'PsycINFO' database and CAS, and is the official journal of The International Neuropsychiatric Association (INA). The manuscript management system is completely online and includes a very quick and fair peer-review system, which is all easy to use. Visit http://www.dovepress.com/testimonials.php to read real quotes from published authors.

\footnotetext{
Submit your manuscript here: http://www.dovepress.com/neuropsychiatric-disease-and-treatment-journal
} 